\title{
Linear Problems of Optimal Control of Fuzzy Maps
}

\author{
Andrej V. PLOTNIKOV ${ }^{1}$, Tatyana A. KOMLEVA ${ }^{2}$ \\ ${ }^{1}$ Department of Applied Mathematics, Odessa State Academy of Civil Engineering and Architecture, \\ Odessa, Ukraine \\ ${ }^{2}$ Department of Mathematics, Odessa State Academy of Civil Engineering and Architecture, \\ Odessa, Ukraine \\ Email:\{a-plotnikov,t-komleva\}@ukr.net
}

\begin{abstract}
In the present paper, we show the some properties of the fuzzy R-solution of the control linear fuzzy differential inclusions and research the optimal time problems for it.
\end{abstract}

Keywords: fuzzy differential inclusions, control problems

\section{Introduction}

The first study of differential equations with multivalued right-hand sides was performed by A. Marchaud [1,2] and S.C. Zaremba [3]. In early sixties, T. Wazewski [4,5], A.F. Filippov [6] obtained fundamental results on existence and properties of the differential equations with multivalued right-hand sides (differential inclusions). One of the most important results of these articles was an establishment of the relation between differential inclusions and optimal control problems, that promoted to develop the differential inclusion theory [7].

Considering of the differential inclusions required to study properties of multivalued functions, i.e. an elaboration the whole tool of mathematical analysis for multivalued functions [8-10].

Simultaneously there were appeared papers [11-14] which used Hukuhara derivative $[9,10]$ of multivalued function for investigation of differential equations with multivalued right-hand sides and solutions.

In works $[15,16]$ annotate of an R-solution for differential inclusion is introduced as an absolutely continuous multivalued function. Various problems for the R- solution theory were regarded in [17-22].

The basic idea for a development of an equation for $\mathrm{R}$-solutions (integral funnels ) is contained in [23].

Here the equation was considered as a particular case of an approximated equation in a metric space. Approximated equations make possible to exclude an differentiation operation and there by to avoid linearity requirement for a solution space and to consider differential equations in linear metric spaces, equations with multivalued solutions and dynamical systems in nonlinear metric spaces by unified positions [14,20,23,24].

Therefore, an approximated equation is a quasidifferential equation for determination of the dynamical system in a metric space.
The theory of mutational equations in metric spaces, which deals with multivalued trajectories (pipers) and trajectories in nonlinear spaces has been developed in [25].

As well as in [23] it is taken an approach that does not use a derivative in explicit form for description in nonlinear metric spaces, while in [25] analogous results are obtained by construction "differential calculus" in nonlinear metric spaces.

Moreover in [23] quasidifferential equations were considered for locally compact spaces, while in [20] for complete metric space .

In the last years there has been forming new approach to control problems of dynamic systems, which foundation on analysis of trajectory bundle but not separate trajectories. The section of this bundle in any instant is some set and it is necessary to describe the evolution of this set. Obtaining and research dynamic equations of sets there is important problem in this case. The metric space of sets with the Hausdorff metric is natural space for description dynamic of sets. In theory of multivalued maps definitions on derivative as for single-valued maps is impossible because space of sets is nonlinear. This bound possibility description dynamic sets by differential equations. Therefore, the control differential equations with set of initial conditions [26-28], the control differential inclusions [29-40], the control differential equations with Hukuhara derivative [14] and the control quasidifferential equations $[14,40,41]$ use for it.

In recent years, the fuzzy set theory introduced by Zadeh [42] has emerged as an interesting and fascinating branch of pure and applied sciences. The applications of fuzzy set theory can be found in many branches of regional, physical, mathematical, differential equations, and engineering sciences. Recently there have been new advances in the theory of fuzzy differential equations [43-55] and inclusions [50,56-59] as well as in the theory of control fuzzy differential equations [60-62] and 
inclusions [63-65].

In this article we consider the some properties of the fuzzy R-solution of the control linear fuzzy differential inclusions and research the optimal time problems for it.

\section{The Control Differential inclusions}

\subsection{The Fundamental Definitions and Designations}

Let $\operatorname{comp}\left(R^{n}\right)\left(\operatorname{conv}\left(R^{n}\right)\right)$ be a set of all nonempty (convex) compact subsets from the space $R^{n}$,

$$
h(A, B)=\min _{r \geq 0}\left\{S_{r}(A) \supset B, S_{r}(B) \supset A\right\}
$$

be Hausdorff distance between sets $A$ and $B, S_{r}(A)$ is $r$-neighborhood of set $A$.

Let $E^{n}$ be the set of all $u: R^{n} \rightarrow[0,1]$ such that u satisfies the following conditions:

1) $u$ is normal, that is, there exists an $x_{0} \in R^{n}$ such that $u\left(x_{0}\right)=1$;

2) $u$ is fuzzy convex, that is,

$$
u(\lambda x+(1-\lambda) y) \geq \min \{u(x), u(y)\}
$$

3) for any $x, y \in R^{n}$ and $0 \leq \lambda \leq 1$;

4) $u$ is upper semicontinuous,

5) $[u]^{0}=\operatorname{cl}\left\{x \in R^{n}: u(x)>0\right\}$ is compact.

If $u \in E^{n}$, then $u$ is called a fuzzy number, and $E^{n}$ is said to be a fuzzy number space. For $0<\alpha \leq 1$, denote

$$
[u]^{\alpha}=\left\{x \in R^{n}: u(x) \geq \alpha\right\} .
$$

Then from 1)-4), it follows that the $\alpha$-level set $[u]^{\alpha} \in \operatorname{conv}\left(R^{n}\right)$ for all $0 \leq \alpha \leq 1$.

Theorem 1 (Negoita and Ralescu [66]). If $u \in E^{n}$, then

1) $[u]^{\alpha} \in \operatorname{conv}\left(R^{n}\right)$ for all $\alpha \in[0,1]$;

2) $[u]^{\alpha} \subset[u]^{\beta}$ for $0 \leq \alpha<\beta \leq 1$;

3) If $\left\{\alpha_{k}\right\} \subset[0,1]$ is a decreasing sequence converging to $\alpha>0$ then $[u]^{\alpha}=\bigcap_{k \geq 1}[u]^{\alpha_{k}}$

Conversely, if $\left\{A^{\alpha}: 0 \leq \alpha \leq 1\right\}$ is a family of convex compact subsets of $R^{n}$ satisfying 1)-3), then $[u]^{\alpha}=A^{\alpha}$ for $0<\alpha \leq 1$ and $[u]^{0}=\overline{\bigcap_{0<\alpha \leq 1} A^{\alpha}} \subset A^{0}$.

If $g: R^{n} \times R^{n} \rightarrow R^{n}$ is a function, then using Zadeh's extension principle we can extend $\tilde{g}$ to $E^{n} \times E^{n} \rightarrow E^{n}$ by the equation

$$
\tilde{g}(u, v)(z)=\sup _{z=g(x, y)} \min \{u(x), v(y)\} .
$$

It is well known that

$$
[\widetilde{g}(u, v)]^{\alpha}=g\left([u]^{\alpha},[v]^{\alpha}\right)
$$

for all $u, v \in E^{n}, 0 \leq \alpha \leq 1$ and continuous function $g$.

Further, we have

$$
[u+v]^{\alpha}=[u]^{\alpha}+[v]^{\alpha},[k u]^{\alpha}=k[u]^{\alpha},
$$

where $k \in R$.

Define $D: E^{n} \times E^{n} \rightarrow[0, \infty)$ by the relation

$$
D(u, v)=\sup _{0 \leq \alpha \leq 1} h\left([u]^{\alpha},[v]^{\alpha}\right),
$$

where $h$ is the Hausdorff metric defined in $\operatorname{comp}\left(R^{n}\right)$.

Then $D$ is a metric in $E^{n}$.

Further we know that [67]:

1) $\left(E^{n}, D\right)$ is a complete metric space,

2) $D(u+w, v+w)=D(u, v)$ for all $u, v, w \in E^{n}$,

3) $D(\lambda u, \lambda v)=|\lambda| D(u, v)$ for all $u, v \in E^{n}$ and $\lambda \in R$.

It can be proved that

$$
D(u+v, w+z) \leq D(u, w)+D(v, z)
$$

for $u, v, w, z \in E^{n}$.

Definition 1. A mapping $F:[0, T] \rightarrow E^{n}$ is strongly measurable if for all $\alpha \in[0,1]$ the set-valued map $F_{\alpha}:[0, T] \rightarrow \operatorname{conv}\left(R^{n}\right)$ defined by $F_{\alpha}(t)=[F(t)]^{\alpha}$ is Lebesgue measurable.

Definition 2. A mapping $F:[0, T] \rightarrow E^{n}$ is said to be integrably bounded if there is an integrable function $h(t)$ such that $\|x(t)\| \leq h(t)$ for every $x(t) \in F_{0}(t)$.

Definition 3. The integral of a fuzzy mapping $F:[0, T] \rightarrow E^{n}$ is defined levelwise by $\left[\int_{0}^{T} F(t) d t\right]^{\alpha}$ $=\int_{0}^{T} F_{\alpha}(t) d t$. The set of all $\int_{0}^{T} f(t) d t$ such that $f:[0, T] \rightarrow R^{n}$ is a measurable selection for $F_{\alpha}$ for all $\alpha \in[0,1]$.

Definition 4. A strongly measurable and integrably bounded mapping $F:[0, T] \rightarrow E^{n}$ is said to be integrable over $[0, T]$ if $\int_{0}^{T} F(t) d t \in E^{n}$.

Note that if $F:[0, T] \rightarrow E^{n}$ is strongly measurable and integrably bounded, then $F$ is integrable. Further if 
$F:[0, T] \rightarrow E^{n}$ is continuous, then it is integrable.

Theorem 2. [43]. Let $F, G:[0, T] \rightarrow E^{n}$ be integrable and $c \in[0, T], \lambda \in R$. Then
a) $\int_{0}^{T} F(t) d t=\int_{0}^{c} F(t) d t+\int_{c}^{T} F(t) d t$;
b) $\int_{0}^{T} F(t)+G(t) d t=\int_{0}^{T} F(t) d t+\int_{0}^{T} G(t) d t$;
c) $\int_{0}^{T} \lambda F(t) d t=\lambda \int_{0}^{T} F(t) d t$
d) $D(F, G)$ is integrable;
e) $D\left(\int_{0}^{T} F(t) d t, \int_{0}^{T} G(t) d t\right) \leq \int_{0}^{T} D(F(t), G(t)) d t$.

Consider the following control linear fuzzy differential inclusions

$$
\dot{x} \in A(t) x+G(t, w), x\left(t_{0}\right)=x_{0},
$$

and the following nonlinear fuzzy differential inclusions

$$
\dot{x} \in F(t, x, w), \quad x\left(t_{0}\right)=x_{0},,
$$

where $\dot{x}$ means $d x / d t ; t \in R_{+}$is the time; $x \in R^{n}$ is the state; $w \in R^{m}$ is the control; $A(t)$ is $(\mathrm{n} \times \mathrm{n})$ - dimensional matrix-valued function; $G: R_{+} \times R^{m} \rightarrow E^{n}$, $F: R_{+} \times R^{n} \times R^{m} \rightarrow E^{n}$ are the set-valued functions.

Let

$$
W: R_{+} \rightarrow \operatorname{conv}\left(R^{m}\right)
$$

be the measurable multivalued map.

Definition 5. Set $L W$ of all single-valued branches of the multivalued map $W(\cdot)$ is the set of the possible controls.

Obviously, the control fuzzy differential inclusion (2) turns into the ordinary fuzzy differential inclusion

$$
\dot{x} \in \Phi(t, x), x\left(t_{0}\right)=x_{0},
$$

if the control $\tilde{w}(\cdot) \in L W \quad$ is fixed and $\Phi(t, x) \equiv$. $F(t, x, \widetilde{w}(t))$

The fuzzy differential inclusions (3) has the fuzzy R-solution, if right-hand side of the fuzzy differential inclusion (3) satisfies some conditions [59].

Let $X(t)$ denotes the fuzzy R-solution of the differential inclusion (3), then $X(t, w)$ denotes the fuzzy R-solution of the control differential inclusion (2) for the fixed $w(\cdot) \in L W$.

Definition 6. The set

$$
Y(T)=\{X(T, w): w(\cdot) \in L W\}
$$

be called the attainable set of the fuzzy system (2).

\subsection{The some properties of the R-solution}

In this section, we consider the some properties of the $\mathrm{R}$-solution of the control fuzzy differential inclusion (1). Let the following condition is true.

\section{Condition A:}

A1. $A(\cdot)$ is measurable on $\left[t_{0}, T\right]$;

A2. The norm $\|A(t)\|$ of the matrix $A(t)$ is integrable on $\left[t_{0}, T\right]$;

A3. The multivalued map $W:\left[t_{0}, T\right] \rightarrow \operatorname{conv}\left(R^{m}\right)$ is measurable on $\left[t_{0}, T\right]$;

A4. The fuzzy map $G: R_{+} \times R^{m} \rightarrow E^{n}$ satisfies the conditions

1) measurable in $t$;

2) continuous in $w$;

A5. There exist $v(\cdot) \in L_{2}\left[t_{0}, T\right]$ and $l(\cdot) \in L_{2}\left[t_{0}, T\right]$ such that

$$
|W(t)| \leq v(t),|G(t, w)| \leq l(t)
$$

almost everywhere on $\left[t_{0}, T\right]$.

A6. The set $Q(t)=\{G(t, w(t)): w(\cdot) \in L W\}$ is compact and convex for almost every $\left[t_{0}, T\right]$,i.e. $Q(t) \in \operatorname{conv}\left(E^{n}\right)$.

Theorem 3. Let the condition $A$ is true.

Then for every $w(\cdot) \in L W$ there exists the fuzzy $R$ - solution $X(\cdot, w)$ such that

1). the fuzzy map $X(\cdot, w)$ has form

$$
X(t, w)=\Phi(t) x_{0}+\Phi(t) \int_{t_{0}}^{t} \Phi^{-1}(s) G(s, w(s)) d s,
$$

where $t \in\left[t_{0}, T\right] ; \Phi(t)$ is Cauchy matrix of the differential equation $\dot{x}=A(t) x$;

2). $X(t, w) \in E^{n}$ for every $t \in\left[t_{0}, T\right]$;

3). the fuzzy map $X(\cdot, w)$ is the absolutely continuous fuzzy map on $\left[t_{0}, T\right]$.

Proof. The proof is easy consequence of the $[32,37,40,59]$ and theorem 1 .

Theorem 4. Let the condition A is true.

Then the attainable set $Y(T)$ is compact and convex.

Proof. The proof is easy consequence of the $[32,37$, 40,59] and theorem 1.

We obtained the basic properties of the fuzzy R-solution of systems (1). Now, we consider the some control 
fuzzy problems.

\section{The Optimal Time Problems}

Consider the following optimal control problem: it is necessary to find the minimal time $T$ and the control $w^{*}(.) \in L W$ such that the fuzzy R-solution of system (1) satisfies one of the conditions:

$$
\begin{gathered}
X\left(T, w^{*}\right) \cap S_{k} \neq \varnothing, \\
X\left(T, w^{*}\right) \subset S_{k}, \\
X\left(T, w^{*}\right) \supset S_{k},
\end{gathered}
$$

where $S_{k} \in E^{n}$ is the terminal set.

Clearly, these time optimal problems are different from the ordinary time optimal problem by that here control object has the volume.

Definition 6. We shall say that the pair $\left(w^{*}(\cdot), X\left(\cdot, w^{*}\right)\right)$ satisfies the maximum principle on $\left[t_{0}, T\right]$, if there exists the vector-function $\psi(\cdot)$, which is the solution of the system

$$
\dot{\psi} \in-A^{T}(t) \psi, \psi(T) \in S_{1}(0)
$$

and the following conditions are true

1) the maximum condition

$$
C\left(\left[G\left(t, w^{*}(t)\right], \psi(t)\right)=\max _{w \in W(t)} C\left([G(t, w)]^{1}, \psi(t)\right)\right.
$$

almost everywhere on $\left[t_{0}, T\right]$;

2) the transversal condition:

a) in the case (5):

$$
C\left(\left[X\left(T, w^{*}\right)\right]^{]}, \psi(T)\right)=-C\left(\left[S_{k}\right]^{1},-\psi(T)\right) ;
$$

b) in the case (6): for all $\alpha \in[0,1]$

$$
C\left(\left[X\left(T, w^{*}\right)\right]^{\alpha}, \psi(T)\right) \leq C\left(\left[S_{k}\right]^{\alpha}, \psi(T)\right)
$$

and there exists $\beta \in[0,1]$ such that

$$
C\left(\left[X\left(T, w^{*}\right)\right]^{\beta}, \psi(T)\right)=C\left(\left[S_{k}\right]^{\beta}, \psi(T)\right) ;
$$

c) in the case (7): for all $\beta \in[0,1]$

$$
C\left(\left[X\left(T, w^{*}\right)\right]^{\alpha},-\psi(T)\right) \leq C\left(\left[S_{k}\right]^{\alpha},-\psi(T)\right)
$$

and there exists $\beta \in[0,1]$ such that

$$
C\left(\left[X\left(T, w^{*}\right)\right]^{\beta},-\psi(T)\right)=C\left(\left[S_{k}\right]^{\beta},-\psi(T)\right) .
$$

Clearly, that there cases of the transversal condition of the maximum principle correspond to the three cases of the time optimal problems.

Theorem 5. (necessary optimal condition). Let the condition A are true and the pair $\left(T, w^{*}(\cdot)\right)$ is optimality. Then the pair $\left(w^{*}(\cdot), X\left(\cdot, w^{*}\right)\right)$ satisfies the maximum principle on $\left[t_{0}, T\right]$.

Proof. The proof is easy consequence of the $[32,37$, 40].

Example. Consider the following control linear fuzzy differential inclusions

$$
\dot{x} \in\left(\begin{array}{cc}
0 & 1 \\
-1 & 0
\end{array}\right) x+w+F, x(0)=0,
$$

where $x=\left(x_{1}, x_{2}\right)^{T}$ is the state; $w=\left(w_{1}, w_{2}\right)^{T} \in W$ $=S_{1}(0)$ is the control; $F \in E^{2}$ is the fuzzy set, where

$$
v(f)=\left\{\begin{array}{ll}
1-4 f_{1}^{2}-9 f_{2}^{2}, & 4 f_{1}^{2}+9 f_{2}^{2} \leq 1 \\
0, & 4 f_{1}^{2}+9 f_{2}^{2}>1
\end{array} .\right.
$$

Consider the following optimal control problem: it is necessary to find the minimal time $T$ and the control $w^{*}(\cdot) \in L W$ such that the fuzzy R-solution of system satisfies of the conditions:

$$
X\left(T, w^{*}\right) \cap S_{k} \neq \varnothing
$$

where $S_{k} \in E^{2}$ is the terminal set such, that

$$
\begin{gathered}
\sigma(x)= \begin{cases}\sqrt{1-\left(x_{1}-2 \pi\right)^{2}-\left(x_{2}-1\right)^{2},} & x \in Q, x_{2} \geq 1 \\
\sqrt{1-\left(x_{1}-2 \pi\right)^{2}} & x \in Q,-1<x_{2}< \\
\sqrt{1-\left(x_{1}-2 \pi\right)^{2}-\left(x_{2}+1\right)^{2}} & x \in Q, x_{2} \leq-1 \\
0 & x \notin Q\end{cases} \\
Q=\left\{\begin{array}{l}
2 \pi-1 \leq x_{1} \leq 2 \pi+1 \\
\left(\begin{array}{l}
x_{1} \\
x_{2}
\end{array}\right) \in R^{2}: \sqrt{1-\left(x_{1}-2 \pi\right)^{2}}-1 \leq x_{2} \leq \\
\leq \sqrt{1-\left(x_{1}-2 \pi\right)^{2}}+1
\end{array}\right\} .
\end{gathered}
$$

Obviously, the optimal pair $T=2 \pi$ and $w^{*}(t)=(\cos (t),-\sin (t))$ satisfy of the conditions of the theorem 5:

1) $\left(w^{*}(t), \psi(t)\right)=C(W, \psi(t))$ for a.e. $t \in[0,2 \pi]$;

2) $C\left(\left[X\left(T, w^{*}\right)\right]^{1}, \psi(T)\right)=-C\left(\left[S_{k}\right]^{1},-\psi(T)\right)$,

where $\psi(t)=(\cos (t),-\sin (t))^{T}$ for a.e. $t \in[0,2 \pi]$,

$$
\begin{gathered}
{\left[X\left(T, w^{*}\right)\right]^{\top}=(T \cos (T),-T \sin (T))^{T}=(2 \pi, 0)^{T},} \\
{\left[S_{k}\right]^{1}=\left\{\left(x_{1}, x_{2}\right)^{T}: x_{1}=2 \pi,-1 \leq x_{2} \leq 1\right\} .}
\end{gathered}
$$




\section{REFERENCES}

[1] A. Marchaud, "Sur Les Champs De Demicones Et Equations Differentielles Du Premier Order," (in French) Bull. Soc. Math. France, No. 62, pp. 1-38, 1934.

[2] A. Marchaund, "Sur les Champs Des Deme-Droites Et Les Equations Differentilles Du Premier Ordre," (in French) Bull. Soc. Math., France, No. 63, pp. 1-38, 1934.

[3] S. C. Zaremba, "Sur une extension de la notion d'equation differentielle," Comptes Rendus Acad. Sc., Paris, No. 199, pp. 1278-1280, 1934.

[4] T. Wazewski, "Systemes De Commande Et Equations Au Contingent," (in French) Bull. Acad. Polon. Sci., Ser. Sci. Math. Astronom. Phys., No. 9, pp. 151-155, 1961.

[5] T. Wazewski, "Sur Une Condition Equivalente E L'equation Au Contingent," (in French) Bull. Acad. Polon. Sci., Ser. Sci. Math. Astronom. Phys., No. 9, pp. 865-867, 1961.

[6] A. F. Filippov, "Classical solutions of differential equations with multi-valued right-hand side," SIAM J. Control, No. 5, pp. 609-621, 1967.

[7] J. P. Aubin and A. Cellina, "Differential inclusions," "Set-valued maps and viability theory," Springer-Verlag, Berlin, Heidelberg, New York, Tokyo, 1984.

[8] J. P. Aubin and H. Frankovska, "Set-valued analysis," Birkhauser, Systems and Control: Fundations and Applications, 1990.

[9] M. Hukuhara, "Sur L'application Semi-Continue Dont La Valeur Est Un Compact Convexe," (in French) Funkcial. Ekvac., No. 10, pp. 43-66, 1967.

[10] M. Hukuhara, "Integration Des Applications Mesurables Dont La Valeur Est Un Compact Convexe," (in French) Funkcial. Ekvac., No. 10, pp. 205-223, 1967.

[11] F. S. De Blasi, and F. Iervolino, "Equazioni Differentiali Con Soluzioni A Valore Compatto Convesso," Boll. Unione Mat.Ital., Vol. 2, No. 4-5, pp. 491-501, 1969.

[12] M. Kisielewicz, "Method of averaging for differential equations with compact convex valued solutions," Rend. Mat., Vol. 9, No. 3, pp. 397-408, 1976.

[13] T. A. Komleva and A. V. Plotnikov, "Differential inclusions with the Hukuhara derivative," Nonlinear Oscil. (N. Y.), Vol. 10, No. 2, pp. 229-245, 2007.

[14] V. A. Plotnikov, A. V. Plotnikov, and A. N. Vityuk, "Differential equations with multivalued right-hand sides," Asymptotics Methods. (in Russian) Odessa, AstroPrint, pp. 354, 1999.

[15] A. I. Panasyuk and V. I. Panasyuk, "Asymptotic optimization of nonlinear control systems," (in Russian) Izdat. Beloruss. Gos. Univ., Minsk, pp. 207, 1977.

[16] A. I. Panasjuk, and V. I. Panasjuk, "An equation generated by a differential inclusion," (in Russian) Mat. Zametki, Vol. 27, No. 3, pp. 429-437, 1980.

[17] A. I. Panasyuk, "Dynamics of sets defined by differential inclusions," (in Russian) Sibirsk. Mat. Zh. Vol. 27, No. 5, pp. 155-165, 206, 1986.
[18] A. I. Panasyuk, "On the equation of an integral funnel and its applications," Differential Equations, Vol. 24, No. 11, pp. 1263-1271, 1988.

[19] A. I. Panasyuk, "Equations of attainable set dynamics. I. Integral funnel equations," J. Optim. Theory Appl., Vol. 64, No. 2, pp. 349-366, 1990. "Equations of attainable set dynamics. II. Partial differential equations," J. Optim. Theory Appl., Vol. 64, No. 2, pp. 367-377, 1990.

[20] A. I. Panasyuk, "Quasidifferential equations in a complete metric space under caratheodory-type conditions," Differential Equations, Vol. 31, No. 6, pp. 901-910, 1995. Differential Equations, Vol. 31, No. 8, pp. 1308-1317, 1995.

[21] A. I. Panasyuk and V. I. Panasyuk, "Asymptotic turnpike optimization of control systems," (in Russian) "Nauka i Tekhnika," Minsk, pp. 296, 1986.

[22] A. A. Tolstonogov, "On an equation of an integral funnel of a differential inclusion," (in Russian) Mat. Zametki, Vol. 32, No. 6, pp. 841-852, 1982.

[23] A. I. Panasyuk, "Quasidifferential equations in a metric space," (in Russian) Differentsial'nye Uravneniya, Vol. 21, No. 8, pp. 1344-1353, 1985.

[24] V. Plotnikov and P. Kitanov, "Continuous dependence of solutions of quasidifferential equations with impulses," Discrete Mathematics and Applications, Blagoevgrad, Vol. 5, pp. 238-245, 1994, Res. Math., "Neofit Rilski" South-West Univ. Publ. House, Blagoevgrad, 1995.

[25] J. P. Aubin, "Mutational equations in metric spaces," SetValued Analysis, Vol. 1, No. 1, pp. 3-46, 1993.

[26] D. A. Ovsyannikov, "Mathematical methods for the control of beams," (in Russian) Leningrad. Univ., Leningrad, pp. $227,1980$.

[27] V. I. Zubov, "Dynamics of controlled systems," (in Russian) Vyssh. Shkola, Moscow, pp. 286, 1982.

[28] V. I. Zubov, "Stability of motion. Lyapunov methods and their application," (in Russian) Vyssh. Shkola, Moscow, pp. 232, 1984.

[29] N. Kikuchi, “On contingent equations," Japan-United States Seminar on Ordinary Differential and Functional Equations, Kyoto, pp. 169-181, 1971, Lecture Notes in Math., Springer, Berlin, Vol. 243, 1971.

[30] S. Otakulov, "A minimax control problem for differential inclusions," Soviet Math. Dokl., Vol. 36, No. 2, pp. 382-387, 1988.

[31] S. Otakulov, "On the approximation of the time-optimality problem for controlled differential inclusions," Cybernet. Systems Anal., Vol. 30, No. 3, pp. 458-462, 1994.

[32] A. V. Plotnikov, "Linear control systems with multivalued trajectories," (in Russian) Kibernetika (Kiev), No. 4, pp. 130-131, 1987.

[33] A. V. Plotnikov, "Compactness of the attainability set of a nonlinear differential inclusion that contains a control," (in Russian) Kibernetika (Kiev), No. 6, pp. 116-118, 1990.

[34] A. V. Plotnikov, "A problem on the control of pencils of 
trajectories," Siberian Math. J., Vol. 33, No. 2, pp. 351-354, 1992.

[35] A. V. Plotnikov, "Two control problems under uncertainty conditions," Cybernet. Systems Anal., Vol. 29, No. 4, pp. 567-573, 1993.

[36] A. V. Plotnikov, "Necessary optimality conditions for a nonlinear problems of control of trajectory bundles," $\mathrm{Cy}$ bern. Syst. Anal., Vol. 36, No. 5, pp. 729-733, 2000.

[37] A. V. Plotnikov, "Linear problems of optimal control of multiple-valued trajectories," Cybern. Syst. Anal., Vol. 38, No. 5, pp. 772-782, 2002.

[38] A. V. Plotnikov and T. A. Komleva, "Some properties of trajectory bunches of controlled bilinear inclusion," Ukr. Math. J., Vol. 56, No. 4, pp. 586-600, 2004.

[39] A. V. Plotnikov and L. I. Plotnikova, "Two problems of encounter under conditions of uncertainty," J. Appl. Math. Mech., Vol. 55, No. 5, pp. 618-625, 1991.

[40] V. A. Plotnikov and A. V. Plotnikov, "Multivalued differential equations and optimal control," Applications of Mathematics in Engineering and Economics (Sozopol, 2000), Heron Press, Sofia, pp. 60-67, 2001.

[41] A. V. Plotnikov, "Controlled quasidifferential equations and some of their properties," Differ. Equations, Vol. 34, No. 10, pp. 1332-1336, 1998.

[42] L. A. Zadeh, "Fuzzy sets," Information and Control, No. 8, pp. 338-353, 1965

[43] O. Kaleva, "Fuzzy differential equations," Fuzzy Sets and Systems, Vol. 24 , No. 3, pp. 301-317, 1987.

[44] O. Kaleva, "The Cauchy problem for fuzzy differential equations," Fuzzy Sets and Systems, Vol. 35, No. 3, pp. 389-396, 1990

[45] O. Kaleva, "The peano theorem for fuzzy differential equations revisited," Fuzzy Sets and Systems, Vol. 98, No. 1, pp. 147-148, 1998.

[46] O. Kaleva, "A note on fuzzy differential equations," Nonlinear Anal., Vol. 64, No. 5, 2006. pp. 895-900.

[47] T. A. Komleva, L. I. Plotnikova, and A. V. Plotnikov, “Averaging of the fuzzy differential equations," Work of the Odessa Polytehnikal University, Vol. 27, No. 1, pp. 185-190, 2007.

[48] T. A. Komleva, A. V. Plotnikov and N. V. Skripnik, "Differential equations with set-valued solutions," Ukrainian Mathematical Journal. ( Springer New York) Vol. 60, No. 10, pp. 1540-1556, 2008.

[49] V. Lakshmikantham, G. T. Bhaskar, and D. J. Vasundhara, "Theory of set differential equations in metric spaces," Cambridge Scientific Publishers, Cambridge, pp. 204, 2006.

[50] V. Lakshmikantham and R. N. Mohapatra, "Theory of fuzzy differential equations and inclusions," Series in Mathematical Analysis and Applications, Taylor \& Francis, Ltd., London, Vol. 6, pp. 178, 2003.
[51] J. Y. Park and H. K. Han, "Existence and uniqueness theorem for a solution of fuzzy differential equations," Int. J. Math. Math. Sci., Vol. 22, No. 2, pp. 271-279, 1999.

[52] J. Y. Park and H. K. Han, "Fuzzy differential equations," Fuzzy Sets and Systems, Vol. 110, No. 1, pp. 69-77, 2000 .

[53] A. V. Plotnikov and N. V. Skripnik, "Differential equations with clear and fuzzy multivalued right-hand sides," (in Russian) Asymptotics Methods, AstroPrint, Odessa, pp. 192,2009

[54] S. Seikkala, "On the fuzzy initial value problem," Fuzzy Sets and Systems, Vol. 24, No. 3, pp. 319-330, 1987.

[55] D. Vorobiev and S. Seikkala, "Towards the theory of fuzzy differential equations," Fuzzy Sets and Systems, Vol. 125 , No. 2, pp. 231-237, 2002.

[56] J. P. Aubin, "Fuzzy differential inclusions," Probl. Control Inf. Theory, Vol. 19, No. 1, pp. 55-67, 1990.

[57] V. A. Baidosov, "Differential inclusions with fuzzy righthand side," Soviet Mathematics, Vol. 40, No. 3, pp. 567-569, 1990.

[58] V. A. Baidosov, "Fuzzy differential inclusions," J. of Appl. Math. and Mechan., Vol. 54, No. 1, pp. 8-13, 1990.

[59] E. Hullermeier, "An approach to modelling and simulation of uncertain dynamical systems," Internat. J. Uncertain. Fuzziness Knowledge-Based Systems, Vol. 5, No. 2, pp. 117-137, 1997

[60] N. D. Phu and T. T. Tung, "Some properties of sheaf-solutions of sheaf fuzzy control problems," Electron. J. Differential Equations, No. 108, pp. 8, 2006. (Electronic) URL: http://ejde.math.txstate.edu.

[61] N. D. Phu and T. T. Tung, "Some results on sheaf-solutions of sheaf set control problems," Nonlinear Anal., Vol. 67, No. 5, pp. 1309-1315, 2007.

[62] N. D. Phu and T. T. Tung, "Existence of solutions of fuzzy control differential equations," J. Sci. Tech. Devel., Vol. 10, No. 5, pp. 5-12, 2007.

[63] T. I. V. Molchanyuk and A. V. Plotnikov, "Linear control systems with a fuzzy parameter," Nonlinear Oscil. (N. Y.) Vol. 9, No. 1, pp. 59-64, 2006.

[64] I. V. Molchanyuk and A. V. Plotnikov, "Necessary and sufficient conditions of optimality in the problems of control with fuzzy parameters," Ukrainian Math. J., Vol 61, No. 3, pp. 457-463, 2009.

[65] V. S. Vasil'kovskaya and A. V. Plotnikov, "Integro differential systems with fuzzy noise," Ukrainian Math. J., Vol. 59, No. 10, pp. 1482-1492, 2007.

[66] C. V. Negoito and D. A. Ralescu, “Applications of fuzzy sets to systems analysis," A Halsted Press Book. John Wiley \& Sons, New York-Toronto, Ont., pp. 191, 1975.

[67] M. L. Puri and D. A. Ralescu, "Fuzzy random variables," J. Math. Anal. Appl., No. 114, pp. 409-422, 1986. 